\title{
Software de Registro de Presença em Sistema Embarcado com Integração Web
}

\author{
Aryclenio X. Barros ${ }^{1}$, Gabriel V. S. da Silva ${ }^{1}$, Rummeniggue R. Dantas ${ }^{1}$ \\ ${ }^{1}$ Escola de Ciências e Tecnologia - Universidade Federal do Rio Grande do Norte \\ (UFRN) \\ Caixa Postal 1524 - 59078-970 - Natal - RN - Brasil \\ aryclenio2010@gmail.com, gvinicius178@gmail.com, rudsondant@gmail.com
}

\begin{abstract}
The following article seeks to propose a software based on an embedded system connected to a database that performs the real time recording of the presence of users by reading radio frequency emitting cards. The hardware can be docked to any port, providing enough processing for dynamic reading and logging. The data analysis of this system is performed by a web page built in Javascript that performs multiple registry connections and has login security, being highly scalable to business standards. The main goal of the program is to eliminate paper expenses in the presence register of educational and business institutions, offering a more sustainable character to this branch and eliminating possible signature fraud.
\end{abstract}

Resumo. O artigo a seguir busca propor um software baseado em um sistema embarcado conectado a um banco de dados que realiza o registro em tempo real de presença de usuários por meio da leitura de cartões emissores de radiofrequência. O hardware pode ser acoplado a qualquer porta, oferecendo processamento suficiente para a leitura e armazenamento dinâmicos. A análise de dados deste sistema é realizada por uma página web construída em Javascript que realiza múltiplas conexões de registro e possui segurança de login, sendo altamente escalável a padrões empresariais. O programa tem como sua vertente principal eliminar os gastos com papel no registro de presença de instituições educacionais e empresariais, oferecendo um caráter mais sustentável a este ramo e eliminando possíveis fraudes de assinatura.

\section{Introdução}

Com a ascensão da tecnologia, muitas empresas e polos universitários passam a demandar otimizações computacionais para os mais variados quesitos, buscando um produto de fácil implementação e que seja aplicável a solução de um problema com o mínimo de custo. Situações como em [1] demonstram a necessidade de se introduzir tecnologia a fim de automatizar e otimizar, neste caso, o registro de fluxo de pessoas.

A fim de facilitar e inovar neste quesito para as mais variadas aplicações foi desenvolvido um projeto baseado na lógica de sistemas embarcados (embedded systems) que segundo Barros (2010) [2], pode ser definido como um software computacional especializado que faz parte de uma máquina maior. O método desenvolvido para esta aplicação tem como objetivo registrar a presença através da leitura e identificação por radiofrequência (RFID), dos códigos presentes nos mais variados cartões que utilizam a tecnologia de NFC (Near Field Communication) para captar as radiofrequências a curta distância.

Como o resultado final se trata de uma página web foram utilizadas as 
linguagens HyperText Markup Language (HTML) e Cascading Style Sheets (CSS) para a criação da interface do sistema e o JavaScript (JS) como linguagem responsável pela aplicação web e C++ para a programação do Hardware.

\section{Trabalhos Relacionados}

Há muitos projetos que utilizam tecnologias semelhantes ao IDbox, como em [3] onde foi utilizada a mesma tecnologia de leitura RFID porém fazendo uso da placa Arduíno, que tem severas limitações e não possui conexão direta com a web. Apesar do banco de dados ser muito bem estruturado e os elementos serem obtidos através da porta USB, integrar isso com um sistema de registro que possa ser acessado através da internet se torna bem mais complexo levando ao uso de outras alternativas como em [4] onde uma memória local é acoplada à placa de processamento para armazenar informações. Foi buscado com o software proposto não depender de estruturas de servidores locais e fazer uso do armazenamento em nuvem. Em [5], apesar do banco de dados ser bem estruturado, o autor faz uso do Ethernet Shield, uma placa acoplada ao Arduíno com entrada Ethernet (RJ-45), que tem um custo consideravelmente elevado, tornando o projeto complexo em vertentes financeiras e exigindo conexão cabeada constante. A IDbox faz uso da placa ESP8266, consideravelmente menor que as mencionadas anteriormente, mais acessível e com integração $\mathrm{Wi}-\mathrm{Fi}$, promovendo mobilidade do hardware e comunicação em tempo real na presença de conexão.

\section{Desenvolvimento}

A IDbox tem como base um software computacional independente e encarregado de executar funções pré determinadas, conhecido na área da computação como System on Chip (SoC). Segundo Rajsuman (2000) [6] um SoC utiliza-se de um circuito integrado que promove alto desempenho para uma aplicação designada. O objetivo final do projeto necessita de um sistema de bom desempenho e possibilidade de conexão à internet e por isso, foi utilizado a placa ESP8266, que possui em sua arquitetura uma placa de conexão WiFi. O dispositivo se assemelha ao funcionamento da placa Arduíno, porém com um tamanho menor em comprimento $(5,1 \mathrm{~cm})$, um poder maior de processamento e um baixo custo.

Para consultar e registrar novos usuários se faz necessário um banco de dados que armazene essas informações. Nesse projeto foi usado o Google Firebase, este Backend as a Service (Baas) funciona de forma simples com fácil usabilidade e conexão, necessitando apenas de uma conta na Google para ter acesso. Na sua forma gratuita o Firebase fornece recursos suficientes para criação desse projeto em pequena escala de forma satisfatória, oferecendo integrações nativas com aplicativos mobile e plataformas web, além de uma biblioteca para comunicação na plataforma Arduino IDE.

\subsection{Hardware}

Com um hardware eficiente é possível integrar o sistema embarcado para trabalhar com fins específicos através da conexão de sensores. Foram utilizados na construção do projeto 2 sensores específicos: MFRC522 que realiza a leitura de dispositivos que emitem radiofrequência, retornando um vetor de dados binário que é convertido via código para uma chave hexadecimal e o DS1302 (Datasheet 1302) que realiza o armazenamento da hora e data atuais. Por fim, um Buzzer é acoplado para emitir sinais sonoros em determinadas ocasiões descritas pelo programador do sistema. O circuito descrito na Figura 1 demonstra a ligação esquemática de todos os componentes descritos acima e o modelo feito em impressora 3D que hospeda os mesmos. 

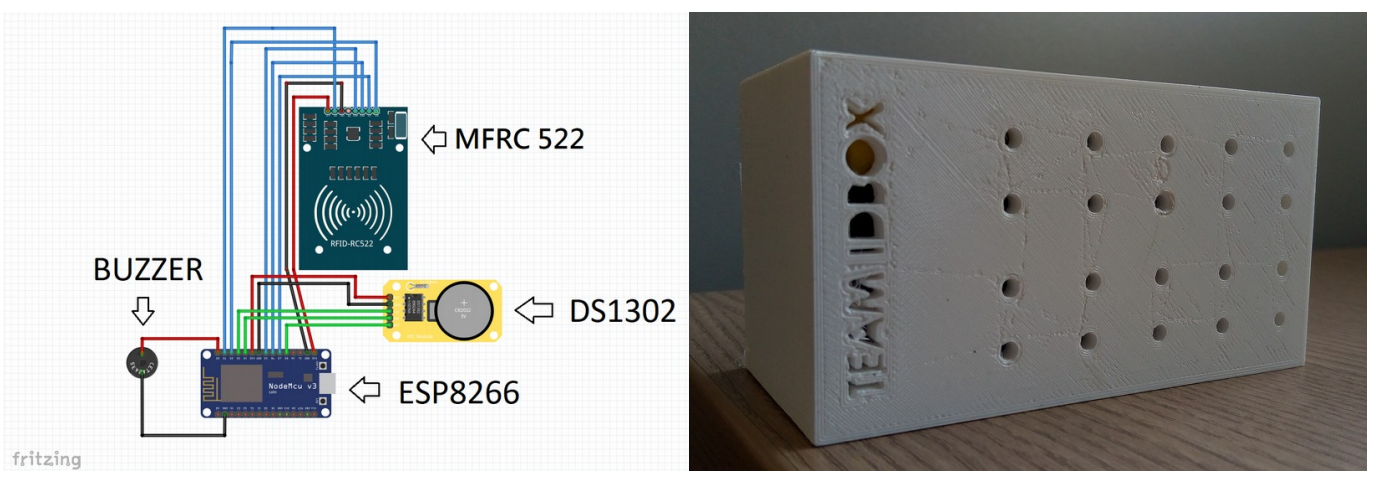

Figura 1. Esquema de circuito e modelo impresso da IDbox.

A chave hexadecimal retornada pelo sensor é única para cada dispositivo lido, onde, combinada com a hora atual da leitura retornada pelo DS1302, é concatenada em um vetor de caracteres e armazenada para registro.

\subsection{Software}

Após a leitura das informações, o vetor de caracteres armazenado é devidamente registrado. Dependendo da quantidade de elementos é possível utilizar Sistemas de Gerenciamento de Banco de Dados (SGBD) mais expansivos como o MySQL. Em nossos testes realizados com o registro de presença, utilizamos um fluxo de usuários menor, que não exige um armazenamento grande, por isso, foi escolhido o Google Firebase, que utiliza o Realtime Database (Banco de Dados em Tempo Real) conseguindo ler e escrever informações de forma mais rápida e eficiente em plataformas distintas de forma simultânea por meio do armazenamento em nuvem.

O dado armazenado é dividido em duas principais vertentes, os elementos básicos do usuário a serem mostrados em tempo real e o armazenamento de logs que registram toda e qualquer alteração feita pelo banco e que podem ser exportadas para qualquer tipo de arquivo de texto. Com o armazenamento, a experiência de visualização das informações é feita através de uma página web integrada a um sistema de login e visualização em tabela, além de contar com o registro direto de usuários. Tudo isso de forma integrada, realizando leitura e escrita de dados em tempo real.
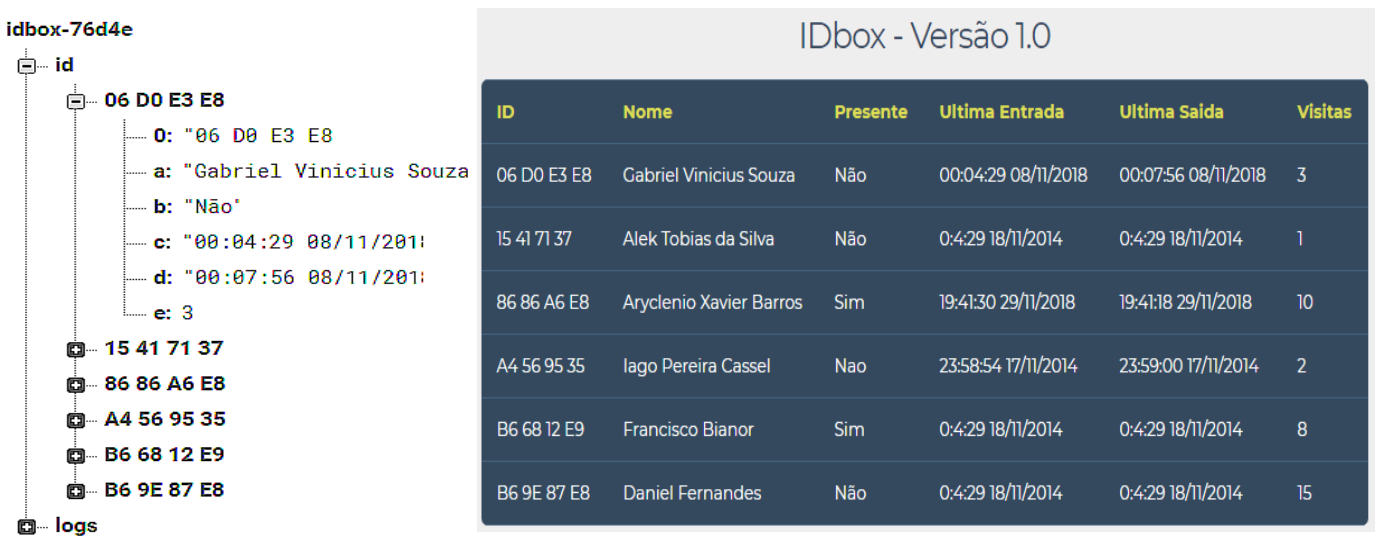

Figura 2. Esquema do banco de dados e interface Web de usuário.

\section{Estudo de Caso}

Foram realizados testes em um laboratório de pesquisa da maior universidade do RN, 
que tem um fluxo diário de 10 a 20 pessoas, localizando o sensor na porta principal e realizando a leitura por meio da carteira de estudante dos integrantes. O sistema se mostrou consistente e registrou bem os dados, conforme visto na figura 2, demonstrando potencial para futuras aplicações em escalas maiores, além de integrações com sensores de presença acoplados na porta de entrada para alertar possíveis passagens de usuários que não fizeram a leitura o cartão.

\section{Conclusão}

Considerando os recursos que o sistema desenvolvido apresenta, várias aplicações são possíveis. De forma simples e arbitrária qualquer aplicação que envolva o armazenamento de dados com tempos marcados pode ser adaptada ou aproveitar-se deste protótipo. Como projeto em desenvolvimento está o registro de presença em salas de aula, onde é possível fazer uso da carteira de estudante, que emite radiofrequência pelo seu chip NFC eliminando assim gastos com papel, que, segundo Virtanen (2013) [7] foram produzidos mais de 360 milhões de toneladas no mundo e boa parte delas acaba em desperdício. O ramo empresarial também poderia utilizar a tecnologia do IDbox para diminuir os custos de implementação de um ponto eletrônico e reduzir também o gasto com papel, sendo assim a continuidade desse projeto almeja trazer mais facilidade e praticidade para as mais diversas aplicações com enfoque atual na sala de aula.

\section{Referencias Bibliográficas}

[1] MOTTA, Thiago Stein. (2016) A experiência de implementação ágil e rápida implantação do sistema de Ponto Eletrônico na UFRGS. In: Workshop de Tecnologia de Informação e Comunicação das Instituições Federais de Ensino Superior (10.: 2016: Gramado). Anais. Gramado,[sn], 2016.

[2] BARROS, Edna; CAVALCANTE, Sérgio. (2010) Introdução aos sistemas embarcados. Artigo apresentado na Universidade Federal de PernambucoUFPE, p. 36.

[3] MOTA, Felipe Vieira. (2018) Tecnologias Embarcadas: Sistema para a captura da frequência de estudantes em dias de aplicação de provas com a utilização da tecnologia RFID. Anais do Encontro Nacional de Pós-Graduação, v. 2, n. 1, p. 132-136.

[4] OLIVEIRA, Rodrigo Morais de; FEITOSA, Marcilio André Félix. (2015) Sistema de Controle de Acesso ao Mestrado em Tecnologia da Energia Utilizando RFID. 2015. 41 f. TCC (Graduação) - Curso de Engenharia da Computação, Escola Politécnica de Pernambuco, Universidade de Pernambuco, Recife.

[5] DEVECCHI, André; SILVA, André Marcos. (2015) Um Sistema de Ponto Eletrônico Digital: Projeto e implementação de hardware e software. 2015. $28 \mathrm{f}$. TCC (Graduação) - Curso de Bacharelado em Ciência da Computação, Faculdade Campo Limpo Paulista, Campo Limpo Paulista.

[6] RAJSUMAN, Rochit. (2000) System-on-a-chip: Design and Test. Artech House, Inc.

[7] VIRTANEN, Yrjo; NILSSON, Sten. (2013) Environmental impacts of waste paper recycling. Routledge. 